\title{
Fermat's Theorem - a Geometrical View
}

\author{
Luis Teia ${ }^{1}$ \\ ${ }^{1}$ Department of Energy Sciences, Lund University Ole Römers Väg 1, M-Building SE-22100 Lund, Sweden \\ Correspondence: Dr. Luis Teia, Department of Energy Sciences, Lund University Ole Römers Väg 1, M-Building SE- \\ 22100 Lund, Sweden. E-mail: luistheya @ gmail.com
}

Received: November 26, 2016 Accepted: January 9, 2017 Online Published: January 23, 2017

doi:10.5539/jmr.v9n1p136

URL: http://dx.doi.org/10.5539/jmr.v9n1p136

\begin{abstract}
Fermat's Last Theorem questions not only what is a triple, but more importantly, what is an integer in the context of equations of the type $x^{n}+y^{n}=z^{n}$. This paper explores these questions in one, two and three dimensions. It was found that two conditions are required for an integer element to exist in the context of the Pythagoras' theorem in 1D, 2D and 3D. An integer must satisfy the Pythagoras' theorem of the respective dimension - condition 1. And, it must be completely successfully split into multiple unit scalars - condition 2. In 1D, the fundamental unit scalar is the line length 1 . All integers in 1D satisfy $x+y=z$, and can be decomposed into multiples of the unit line, hence integers exist and can form 1D triples $(x, y, z)$. In 2D, the fundamental unit scalar is the square side 1. Only some groups of integers (called triples) satisfy $x^{2}+y^{2}=z^{2}$, and can be decomposed into multiples of the unit square, forming 2D triples. In 3D, the fundamental unit scalar is the octahedron side 1. The geometry of the 3D Pythagoras' theorem dictates that $x^{3}+y^{3}=z^{3}$ is governed by octahedrons, validating condition 1 . However, octahedrons with side length integer cannot be completely divided into unit octahedrons (as tetrahedrons appear), invalidating condition 2. Hence, if integers do not exist in the context of the 3D Pythagoras' theorem, then neither do triples. This confirms Fermat's Last Theorem for three dimensions $(n=3)$. The geometrical interdependency between integers in 1D and 2D suggests that all integers of higher dimensions are built, and hence are dependent, on the integers of lower dimensions. This interdependency coupled with the absence of integers in 3D suggests that there are no integers above $n>2$, and therefore there are also no triples that satisfy $x^{n}+y^{n}=z^{n}$ for $n>2$.
\end{abstract}

Keywords: geometry, Fermat, Pythagoras, theorem

\section{Introduction}

Fermat's conjecture, also known as Fermat's Last Theorem (Dickson, 1919), is more than just about triples, it is about the fundamental nature of an integer number, and it's mathematical and geometrical meaning. It raises the philosophical question: What is a unit? In the language of mathematics, a unit is defined by the number 1 . In the language of geometry, a unit is defined by an element of side length one. A perspective of a problem depends on the language we use to observe it. And a change in perspective is often all it takes to see the solution. In mathematics, the manipulation of the number 1 (i.e., summation, subtraction, multiplication, division, etc) gives rise to the whole universe of numbers. Whether it is a complex, real or integer number, they all can be expressed as a manipulation of one or a group of ones (Figure 1). Hence, the number 1 is the fundamental building block of the mathematical universe of numbers.

$$
\text { Complex : } i=\sqrt{-1} \quad ; \quad \text { Real : } 1,2=1+\frac{1+1}{1+1+1+1+1+1+1+1+1+1} \quad ; \text { Integer }: 2=1+1
$$

Figure 1. Examples of numbers built mathematically with 1.

Geometry, the field of knowledge dealing with spatial relationships, predates modern mathematics (Cooke, 2005), being later revolutionized by the book Elements (Euclid, 300BC) into the foundation of contemporary mathematics. Hence, every mathematical operation has a geometrical meaning. For example, mathematical operations with powers 1,2 and 3 translate into geometric operations in one, two, and three dimensions (Figure 2). Visualization of such geometrical operations can be overwhelmingly complex, and hence mathematics is used to bypass such complexity, allowing the manipulation of certain aspects of the underlying geometrical operation.

At the end, a mathematical answer that has some geometrical meaning is obtained. Completeness is sacrificed when changing from a geometrical perspective to mathematical, resulting in an easier and speedier answer. In geometry, the manipulation of the unit element gives rise to the universe of elements, equivalent to the mathematical universe of numbers. A number has different meanings in different dimensions. In the 1D Pythagoras' theorem, i.e. the fundamental process of 


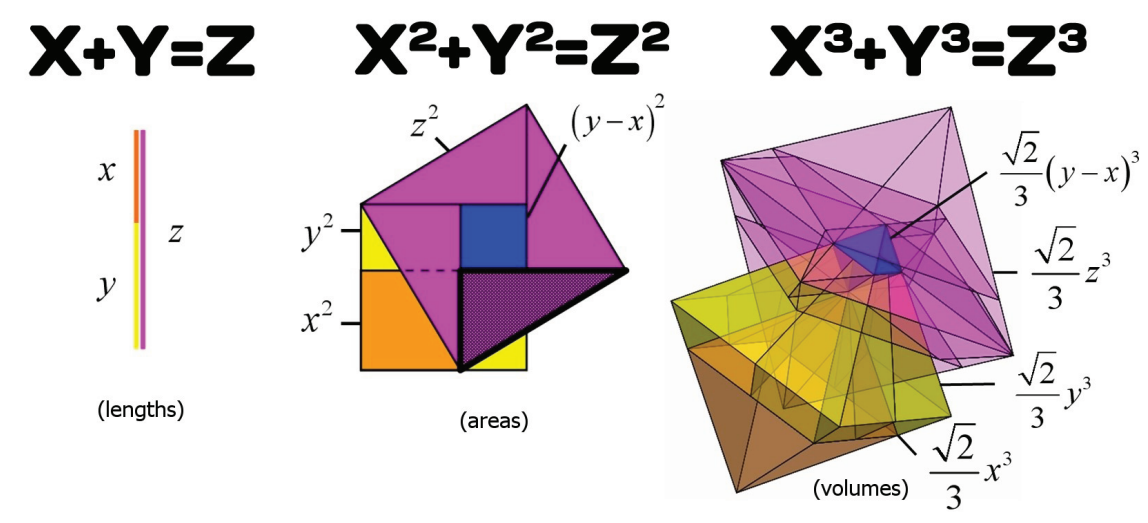

Figure 2. The 1D, 2D and 3D Pythagoras' theorem (Teia, 2016)

summation $x+y=z$, numbers are governed by lines with given lengths. In 2D, numbers are governed by squares with given areas. And in 3D, numbers are governed by octahedrons with given volumes (Teia, 2016). These geometrical units (i.e., line, square and octahedron) establish the foundation for the geometric interpretation of integers. Since triples are aggregates of three integer numbers, this raises the question - how does the mathematical and geometrical meaning of triples evolves from $1 \mathrm{D}$ to $2 \mathrm{D}$, and finally to $3 \mathrm{D}$ ?

\section{Theory}

\subsection{The $1 D$ and 2D Pythagoras' Theorem}

Let us start with the simple one dimensional version of the Pythagoras' theorem. It is governed by line segments, where the geometrical addition of two line segments gives a third, or $x+y=z$ (Figure 3a). This is by definition the mathematical process of summation. One process that transforms the 1D Pythagoras' theorem to $2 \mathrm{D}$ is defined by the following two steps:

Step 1. Rotation by 90 degrees between two lines about the middle point. This gives a right-angled triangle (Figure 3b).

Step 2. Lines are extruded perpendicularly to their length and form squares. The result is the two dimensional version of the Pythagoras' theorem, where the geometric addition of two squares gives a third, or $x^{2}+y^{2}=z^{2}$ (Figure 3c).

The necessity for an integer within a triple to satisfy the geometric part of the Pythagoras' theorem is, from now on, termed Condition 1.

(a)

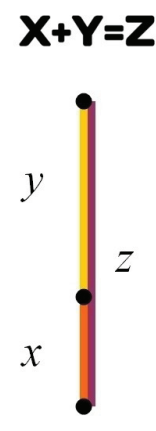

(b)
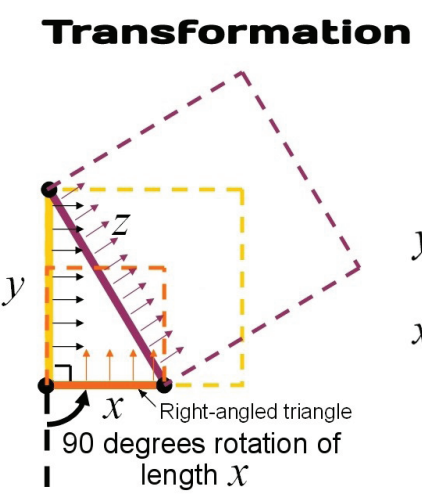

(c)

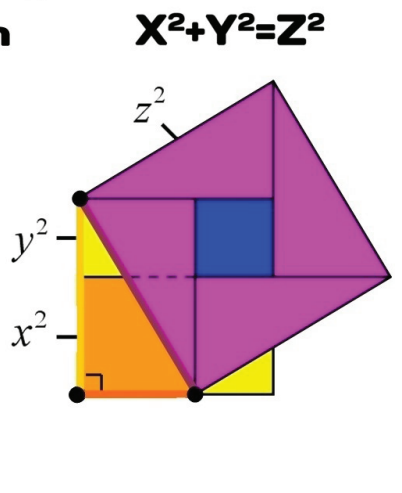

Figure 3. The geometry of (a) $x+y=z$, (b) the transformation, and (c) $x^{2}+y^{2}=z^{2}$

Mathematical numbers are grouped into different categories, like real and integer. Real numbers $(\mathbb{R})$ compose the entire universe of numbers and can be thought of as points on an infinite long number line. They include all the rational numbers, such as fractions and integers (Clapham \& Nicholson, 2014). Integers $(\mathbb{Z})$ are a subgroup of real numbers (Figure 4). An integer is a number that can be written without a fractional component (i.e., 1,2,3). Triples are a set of three integer numbers that satisfy the Pythagoras' theorem, hence a subgroup of integer numbers. Figure 4 shows 
examples of mathematical numbers that belong to these three divisions, and satisfy the 1D and 2D Pythagoras' theorem. Mathematical summations of these numbers and their geometric equivalent are presented.

\section{$\mathbf{X}+\mathbf{Y}=\mathbf{Z}$}

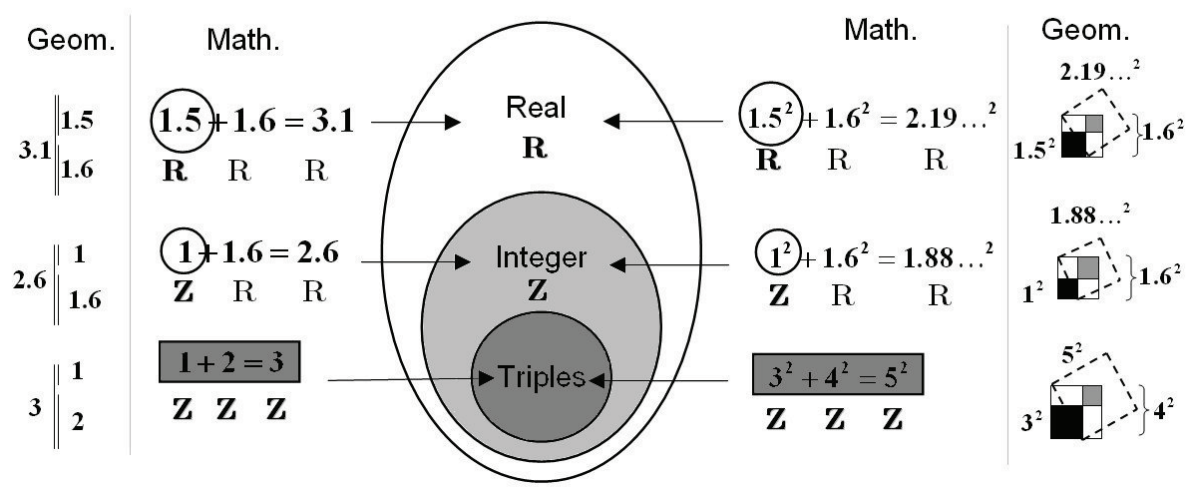

Figure 4. Groups of numbers in the Real, Integer, and Triple domains

Example 1 (Figure 4 left) shows two lines of length real numbers 1.5 and 1.6 give another line of length real number 3.1. Example 2 shows an integer 1 plus a real number 1.6 give another real number 2.6. Finally, example 3 shows a group of integer numbers 1, 2 and 3 that satisfies the 1D Pythagoras' theorem, and hence forms the 1D triple $(1,2,3)$. Similar summations are observed for 2D. The area of two squares $1.5^{2}$ and $1.6^{2}$ gives the area of a third square $2.19 \ldots{ }^{2}$ (Figure 4 right). And, a group of squares $3^{2}, 4^{2}$ and $5^{2}$ satisfies the 2D Pythagoras' theorem, and hence forms the 2D triple $(3,4,5)$.

\subsection{Geometry of $1 D$ and $2 D$ Integers}

The unit line, or line of length 1, is the fundamental geometric scalar that composes all integer elements in the 1D universe (i.e., $2^{1}, 3^{1}, 4^{1}, 5^{1} \ldots N^{1}$, where $N$ is an integer number). As illustrated in Figure $5 \mathrm{a}$, all integer lines are formed by an aggregate chain of unit lines. Likewise, the unit square, or square of side 1, is the fundamental geometric scalar that composes all integer elements in the $2 \mathrm{D}$ universe (i.e., $2^{2}, 3^{2}, 4^{2}, 5^{2} \ldots N^{2}$ ). As illustrated in Figure $5 \mathrm{~b}$, all integer squares are formed by an aggregate chain of unit squares. Generally, one can conclude from Figure 5 that in order for an integer element to exist, it needs to be completely split into multiples of the fundamental unit scalar particular to that dimension (i.e., unit line in 1D or unit square in 2D). The necessity for an integer (in order to be an integer) to be split into a multiple of a fundamental scalar of the respective dimension is from now on termed Condition 2.

(a)

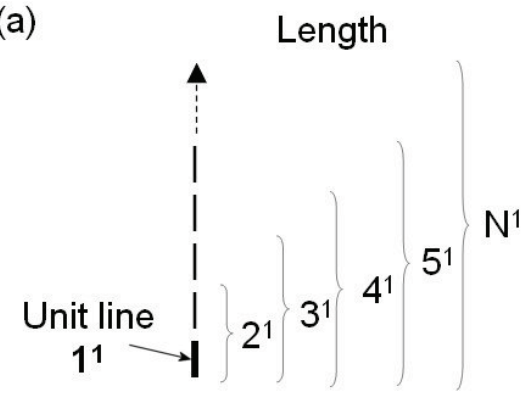

(b)

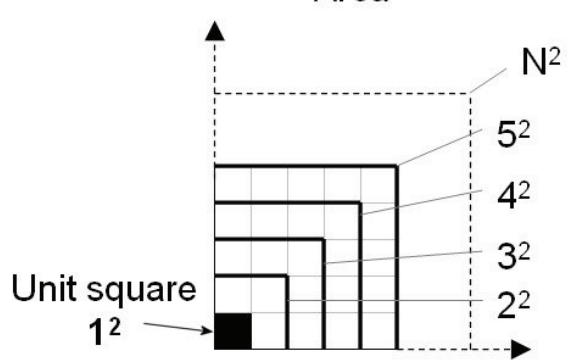

Figure 5. Integers are aggregate chains of unity scalars in (a) one and (b) two dimensions

The geometry of the scalar governs all integers that satisfy the Pythagoras' theorems, and consequently, all triples. The world of $1 \mathrm{D}$ triples is governed by the unit line (e.g., $1+2=3$ ), while for $2 \mathrm{D}$ triples, it is the unit square (e.g., $3^{2}+4^{2}=5^{2}$ ). Figure 6 shows the spiral of triples belonging to the Pythagoras' family, drawn using the central square approach (Teia, 2015), with a grid of unit squares superimposed.

It is seen that all squares that compose $2 \mathrm{D}$ triples (e.g., $3^{2}, 4^{2}, 5^{2}$ ) can be decomposed into a sum of unit squares. One can generalize this and say that all squares composing the integers of the 2D triple $(x, y, z)$ can be expressed geometrically by aggregates of many unit squares, hence satisfying Condition 2. 


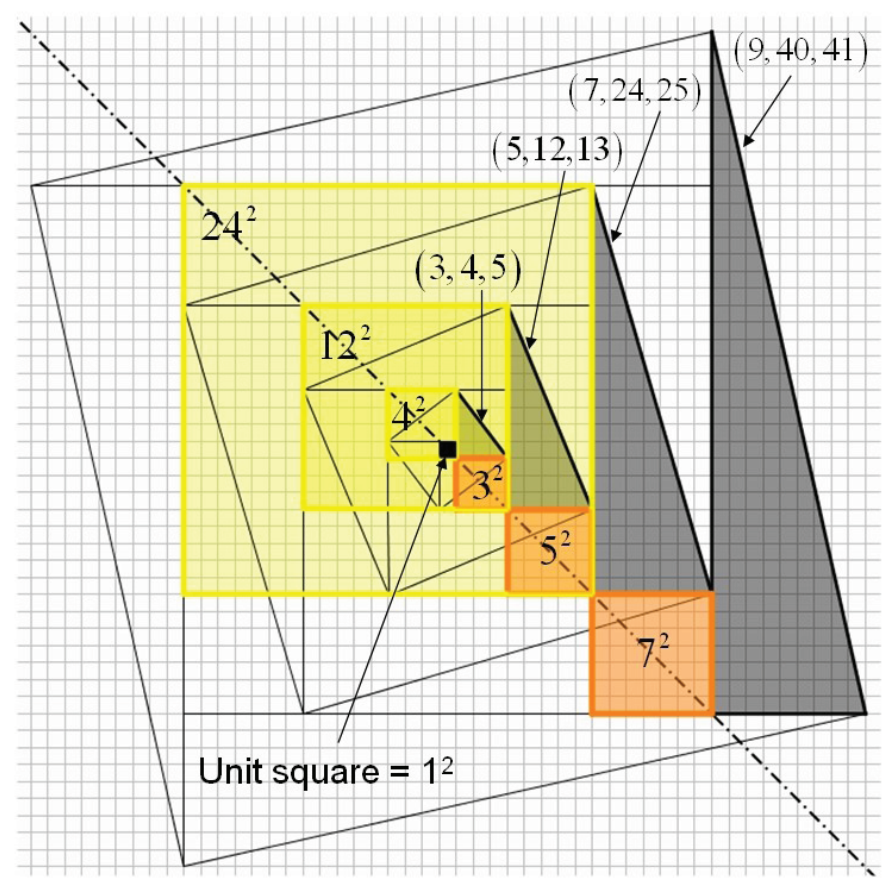

Figure 6. The Pythagoras' family of triples expressed via unit squares (Teia, 2015)

\section{Hypothesis}

A triple only exists, if all integer elements within that triple (e.g., $1^{1}, 2^{1}, 3^{1}$ for $1 \mathrm{D}$, and $3^{2}, 4^{2}, 5^{2}$ for $2 \mathrm{D}$ ) also exist. In turn, an integer element only exits if:

Condition 1. it satisfies the Pythagoras' theorem of the respective dimension,

Condition 2. it can be completely successfully split into multiple unit scalars.

One can therefore hypothesize that integer elements do not exist if either Condition 1 or 2 is not satisfied. By consequence, if the integers do not exist, then the associated triples also do not exist.

\section{Development}

\subsection{Triples Transition from $1 D, 2 D$ to $3 D$}

Integers in 1D and 2D Pythagoras' theorem have been shown to satisfy both conditions. Hence, all integers in 1D form triples (e.g., $1+1=2,1+2=3,1+4=5$ ) [Figure 7 left] and some integers in $2 \mathrm{D}$ form triples (e.g., $3^{2}+4^{2}=5^{2}$, $5^{2}+12^{2}=13^{2}, 7^{2}+24^{2}=25^{2}$ ) [Figure 7 middle]. The aggregate of these triples forms the well known ternary tree of Pythagorean triples (Teia, 2016a). The question that rises then is - do integers in 3D satisfy Conditions 1 and 2 so that triples are possible? [Figure 7 right]. Or, is Fermat's conjecture right, and there are no triples in 3D?

\subsection{The 3D Pythagoras' Theorem}

This is sometimes confused with Fermat's Conjecture. Fermat's Last Theorem is a mathematical conjecture about integer numbers (Singh, 2002), while the 3D Pythagoras' theorem is a mathematical and geometrical proof about real numbers (Teia, 2015a). How does one build the 3D Pythagoras' theorem? This is now briefly explained. It is assumed that the process used to convert the 1D Pythagoras' theorem to 2D, shown previously in Figure 3, also applies from 2D to 3D.

The two steps for converting the 2D Pythagoras' theorem into 3D are:

Step 1. Rotation of the Z-square by 90 degrees (Figure 8a) forms a central octahedron [in blue] (Figure 8b) just as a central square [in blue] appeared naturally when passing from 1D to 2D (Figure 3). Both the central square (in 2D) and central octahedron (in 3D) are a direct and inherent consequence of the process. Hence, the geometric element that governs the 3D Pythagoras' theorem is an octahedron.

Step 2. Perpendicular extrusion along the axis of symmetry transforms squares into octahedrons. Finally, careful partition of the octahedrons gives the 3D Pythagoras' theorem (Figure 8c), which is equivalent to the 2D Pythagoras' theorem (Figure 8a). This partition is explained in detail in Teia (2015a). 


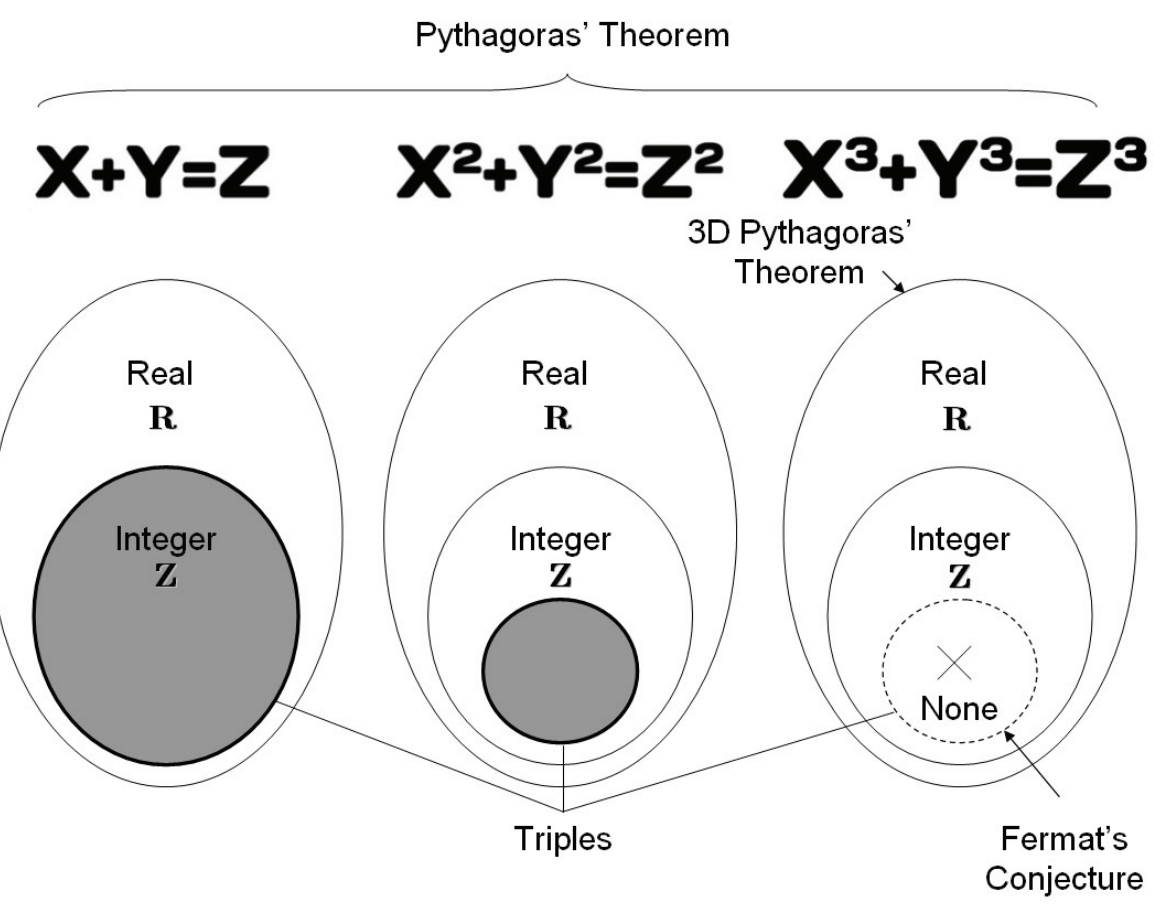

Figure 7. Triples in the world of one, two and three dimensional Pythagoras' theorem

An octahedron satisfies the 3D Pythagoras' theorem, and hence verifies Condition 1. Hence, in order for 3D integers to exist in the Pythagoras' world, octahedrons also need to satisfy Condition 2. The question then is - can an integer octahedron be split into multiple unit octahedrons?

(a)
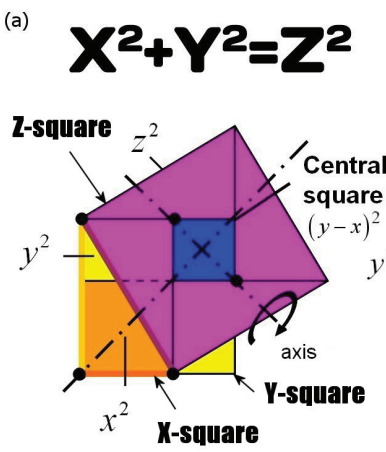

(b)

Transformation

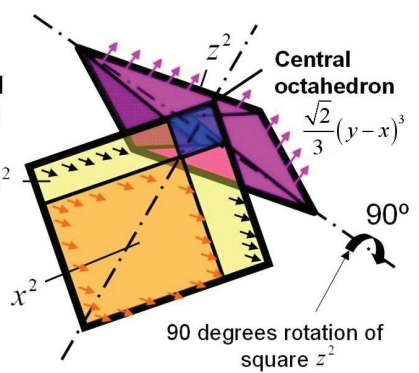

(c)

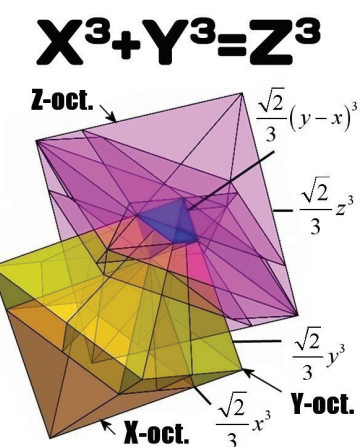

Figure 8. Transformation of the Pythagoras' theorem from 2D to 3D (Teia, 2015a)

\subsection{Geometry of $3 D$ Integers}

The unit scalars in 1D (the unit line) and 2D (the unit square) have shown to correctly represent all integer elements in their respective dimension (Figure 5). For example, in 1D we have the integer $2^{1}$ (the upper case 1 represents the dimension) defined by two unit lines, and in $2 \mathrm{D}$ we have $2^{2}$ defined by four unit squares. Hence, the squares that compose the 2D Pythagoras' theorem can also be split into multiple unit squares (Figure 9a). The question now is - can the 3D Pythagoras' theorem be split into multiple unit octahedrons (Figure 9b)?

Let us try to build, using only unit octahedrons, the first 3D integer after $1^{3}-$ the $2^{3}$. In order to build an integer octahedron with side 2 , it is necessary to link 6 unit octahedrons (Figure 10a). They do not completely satisfy the volume of $2^{3}$, as there are tetrahedral gaps in the middle. Hence, eight additional unit tetrahedrons are required to complete the volume (Figure 10b).

This means that the overall integer octahedron $2^{3}$ is defined by 6 unit octahedrons and 8 unit tetrahedrons (Figure 10c). This can be extrapolated to any integer octahedron $N^{3}$, where $\mathrm{N}$ is an integer. Take, as another example, the integer 
(a)

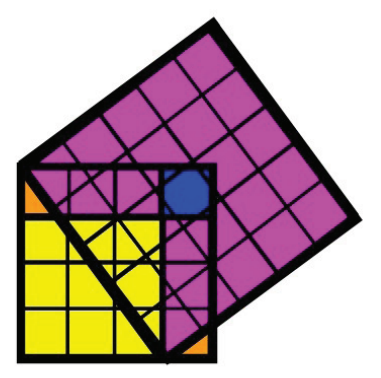

(b)

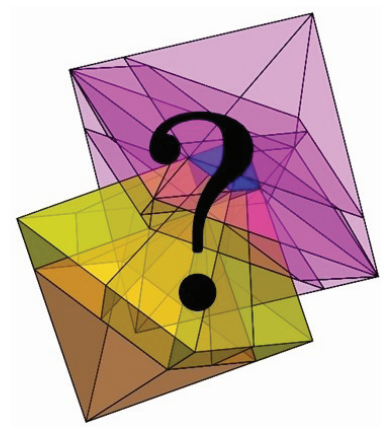

Figure 9. Partition of Pythagoras' theorem into multiple unit scalars (a) 2D and (b) 3D

(a)

\section{Unit Octahedrons}

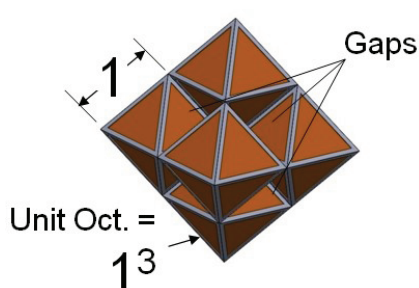

(b)

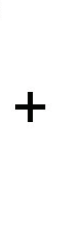

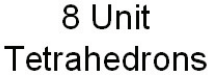

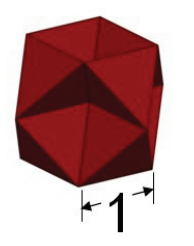

(c)

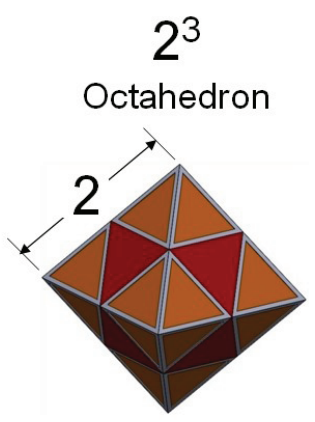

Figure 10. The octahedron $2^{3}$ : (a) unit octahedrons, (b) unit tetrahedrons and (c) all together

octahedron $3^{3}$, which is an extension of the $2^{3}$. The integer $2^{3}$ is composed of 4 unit octahedrons at midplane, and 1 on either side up and down (as shown in Figure 11a). Adding four unit tetrahedrons on either side of the midplane completes the volume. The integer $3^{3}$ (Figure 11b), in turn, is formed by adding to $2^{3}$ a new layer of 9 unit octahedrons at midplane, and 12 unit tetrahedrons on either side. Therefore, the proportion of unit octahedrons to unit tetrahedrons changed to 19 and 32, respectively. This can be extrapolated to an octahedron side integer N. It can be shown that the number of unit octahedrons is $\sum_{i=1}^{N} i^{2}+\sum_{i=1}^{N-1} i^{2}$ and unit tetrahedrons is $2 \sum_{j=1}^{N-1} \sum_{i=1}^{j} 4 i$ (Figure 11c).

(a)

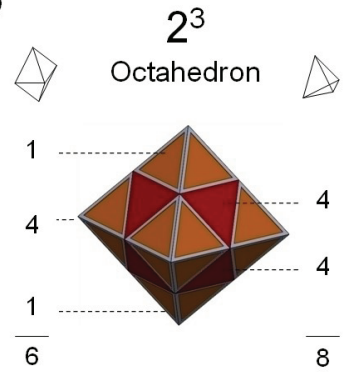

(b)

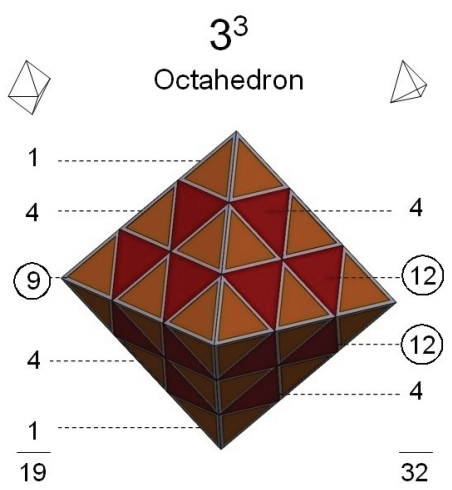

(c)

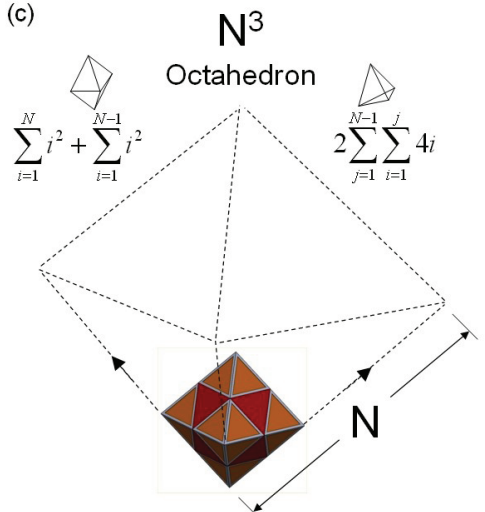

Figure 11. Composition of octahedrons (a) $2^{3}$, (b) $3^{3}$ and (c) $N^{3}$.

Integers are clear multiples of a unit. When a line is solely composed of multiple unit lines, the number it represents is an integer. When a square is solely composed of multiple unit squares, it also represents an integer. An octahedron is not solely composed of multiple unit octahedrons, hence it is not an integer. Therefore, even though octahedrons validate the 3D Pythagoras' theorem, and hence Condition 1, the integers $\left(2^{3}, 3^{3}, \ldots N^{3}\right)$ do not exist in the context of Pythagoras' theorem as they don't satisfy Condition 2, i.e. not completely divisible into multiples of the fundamental geometrical unit. 
This non-compliance extends to the entire universe of 3D integers, and validates the hypothesis that three dimensional integers do not exist within the context of the 3D Pythagoras' theorem. By consequence, if integers do not exist, then neither do their associated triples. This confirms Fermat's Conjecture for three dimensions.

Since integers from different dimensions are dependent on each other - for example, 2D integers are formed from 1D integers (i.e., squares of side 1 are formed from lines of length 1) - it would be expected integers in 3D to be formed from integers in 2D. However, it was shown that 3D integers do not exist within the 3D Pythagoras' theorem, it follows from this interdimensional dependency that if integers in 3D don't exist, then neither do in 4D (as they would be dependent on the 3D ones for geometrical construction) or any other higher dimension. This can be generaly stated as, integers within the Pythagoras' theorem for any dimension above 2 (i.e., for $n>2$ where $\mathrm{n}$ is the number of dimensions) do not exist. If integers do no exist, then neither do their respective triples. Ultimately, this means that triples for dimensions $n>2$ do not exist in the domain of the Pythagoras' equation $x^{n}+y^{n}=z^{n}$.

\section{Conclusion}

Fermat's Last Theorem is more than just about triples, it is about the fundamental nature of an integer, and it's mathematical and geometrical expression as a multiple of a unit. What are the conditions that define an integer in the context of the Pythagoras' theorem? One must first study how the Pythagoras' theorem is formed in all its dimensions. The 1D Pythagoras' theorem transforms to 2D in two steps: a 90 degree rotation, and a perpendicular extrusion. Condition 1 dictates that an integer within a triple must satisfy the geometrical part of the Pythagoras' theorem. All integers are formed by an aggregate chain of the fundamental unit specific to that dimension. That is, $1 \mathrm{D}$ integers like $2^{1}, 3^{1}$ are defined in the Pythagoras' theorem by unit lines, and 2D integers like $2^{2}, 3^{2}$ by unit squares. Condition 2 dictates that in order for an integer to be an integer, it must be successfully split into multiples of the fundamental geometric unit of the corresponding dimension. Condition 1 and 2 are valid in both 1D and 2D. The 2D Pythagoras' theorem transforms to 3D using the same steps as before. An octahedron emerges naturally during the process. This is the element that governs the geometry of the 3D Pythagoras' theorem, and verifies Condition 1. However, an integer octahedron cannot be built from aggregates of unit octahedrons, as gaps appear in the form of tetrahedrons, and hence Condition 2 is not verified. This means that the fundamental element that governs the 3D Pythagoras' theorem - the octahedron - cannot be used to construct 3D integers. Therefore, the very notion of integer elements like $2^{3}, 3^{3}$ do not exist in the three dimensional Pythagoras' world, and consequentially neither do triples. This confirms Fermat's Last Theorem for three dimensions. The geometrical interdependency between integers in 1D and 2D suggests that all integers of higher dimensions are built, and hence are dependent, on the integers of lower dimensions. This interdependency coupled with the absence of integers in 3D suggests that there are no integers above $n>2$, and therefore there are also no triples that satisfy $x^{n}+y^{n}=z^{n}$ for $n>2$.

\section{References}

Clapham, C., \& Nicholson, J. (2014). The Concise Oxford Dictionary of Mathematics, 5th ed., Oxford University Press.

Cooke, R. (2005), The History of Mathematics, New York Wiley-Interscience. https://doi.org/10.1002/9781118033098

Dickson, L. E. (1919). History of the Theory of Numbers. (Vol. 2). Diophantine Analysis. New York: Chelsea Publishing.

Euclid (300 BC). Elements. Book 1.

Singh, S. (2002). Fermat's Last Theorem: The Story of a Riddle That Confounded The World's Greatest Minds for 358 Years, published via Amazon.

Teia, L. (2015). Pythagoras' triples explained by central squares, Australian Senior Mathematics Journal, 29, 7-15.

Teia, L. (2015a). $X^{3}+Y^{3}=Z^{3}$ : The Proof, published via Amazon.

Teia, L. (2016). Geometry of the 3D Pythagoras' theorem, Journal of Mathematics Research, 8, 78-84. https://doi.org/10.5539/jmr.v8n6p78

Teia, L. (2016a). Anatomy of the Pythagoras' tree, Australian Senior Mathematics Journal, 30, 38-47.

\section{Copyrights}

Copyright for this article is retained by the author(s), with first publication rights granted to the journal.

This is an open-access article distributed under the terms and conditions of the Creative Commons Attribution license (http://creativecommons.org/licenses/by/4.0/). 